\title{
Attachment of Suspension Cells for TEM Processing
}

\author{
Scott Emrich ${ }^{1}$ and Han Chen ${ }^{2}$
}

${ }^{1}$ Penn State College of Medicine, Pennsylvania, United States, ${ }^{2}$ Penn State College of Medicine, Hummelstown, Pennsylvania, United States

The first transmission electron microscope (TEM) was constructed in the early 1930s by Ernst Ruska and Max $\mathrm{Knoll}^{[1]}$. TEM has developed our knowledge about the ultrastructure of the cells and tissues. Biological systems are very complex; thus, it is impossible to understand structure-function relationships outside the surrounding context. TEM gives opportunities to visualize an interesting target with surrounding structure when unlabelled surroundings remain hidden at fluorescence sample ${ }^{[2]}$. TEM with conventional preparation is still an essential tool in many fields of tissue and cell biology, as well as in medicine ${ }^{[3]}$. However, TEM analysis of rare cell populations, especially cells in suspensions such as primary cells from bone marrow and blood, remains limited due to the requirement of a high cell number during sample preparation. There are a few different approaches for TEM analysis from scarce samples, but these approaches fail to get significant quantitative data from the limited number of cells.

Here, an alternative approach for sample preparation in TEM studies is described for limited cell populations that enable quantitative analysis. A relatively limited cell number, i.e., 100,000 primary lymphocytes, was successfully used for TEM analysis compared to the millions of cells typically used for TEM studies. In particular, Thermanox coverslips were used to getting suspension cells to stick to the poly-L-lysine treated coverslips surfaces. Clusters of numerous cells were observed in ultrathin sections. The cells had a wellpreserved morphology, and the ultra-structural details of the Golgi complex and several mitochondria were visible. This efficient, easy, and reproducible protocol allows sample preparation from a low cell number and can be used for qualitative and quantitative TEM analysis on rare cell populations from limited biological samples.

The brief protocol of attachment of suspension cells for TEM processing:

1. To coat the entirety of the bottom of a $13 \mathrm{~mm}$ Thermanox coverslip for TEM, you will need $10^{5}$ cells for an even monolayer.

2. Add the poly-L-lysine solution to completely cover the surface of the coverslip. Incubate at room temperature for 30 minutes.

3. Aspirate the poly-L-lysine solution and allow the coverslip to completely air dry.

4. Add the cells to the cell culture dish and ensure that the cell suspension is completely covering the coverslip surface. Keep the dish on a flat surface and allow the cells to settle and stick to the bottom of the coverslip at room temperature or in the incubator for 20-30 minutes. Check the cells under a cell culture microscope to make sure they are well attached. The key for getting suspension cells to stick to coverslip surfaces is the removal of serum. Cells can be kept in any balanced PBS solution for attachment as long as all growth factors and serum have been removed.

5. Using a serological pipette, very slowly remove the solution and add TEM fixation buffer by allowing the solution to slowly run down the side of the dish to avoid disturbing the cells.

6. After primary fixation, start the resin embedding process. 


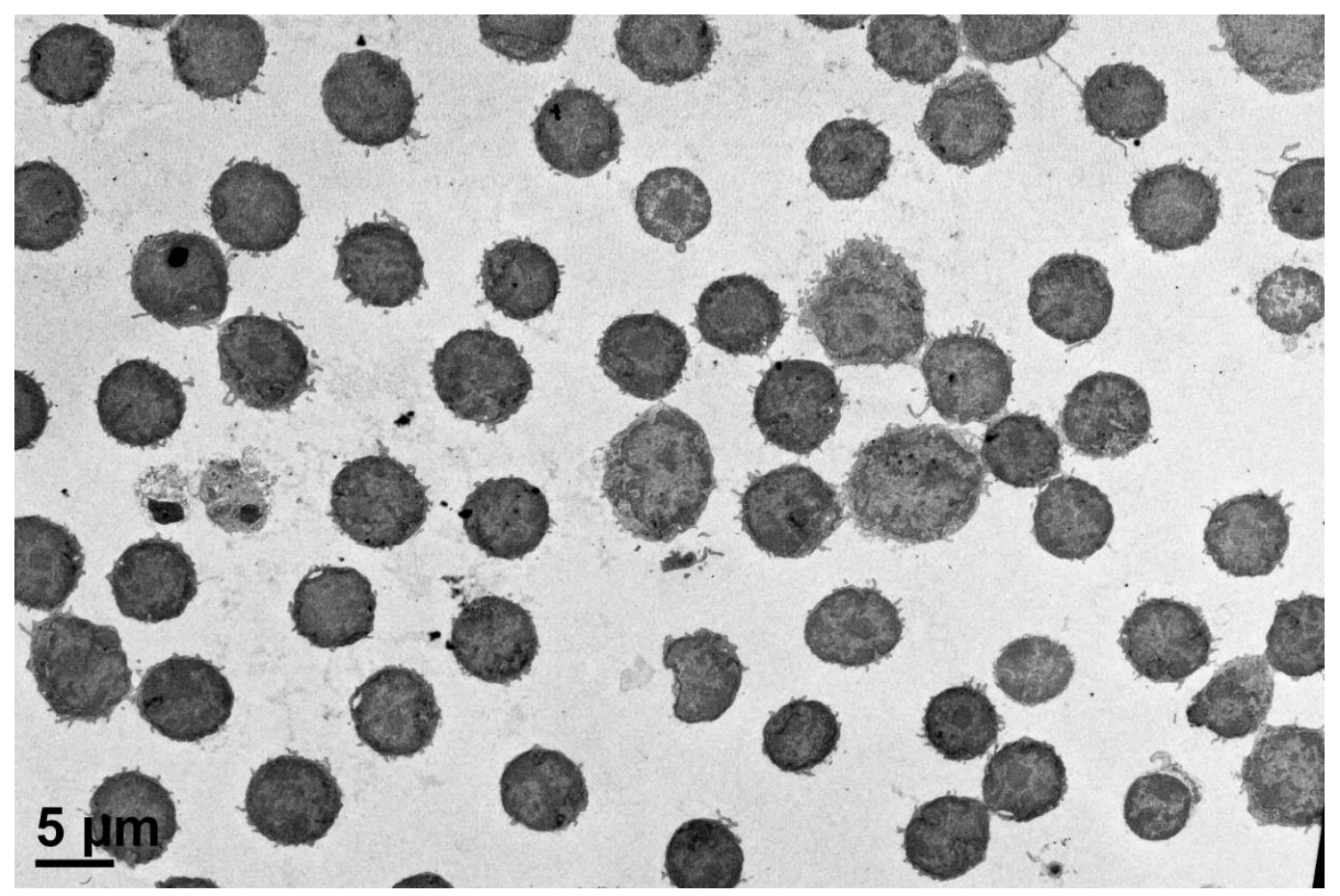

Figure 1. Figure 1: Low magnification image of a representative field. Scale bar: 5 um

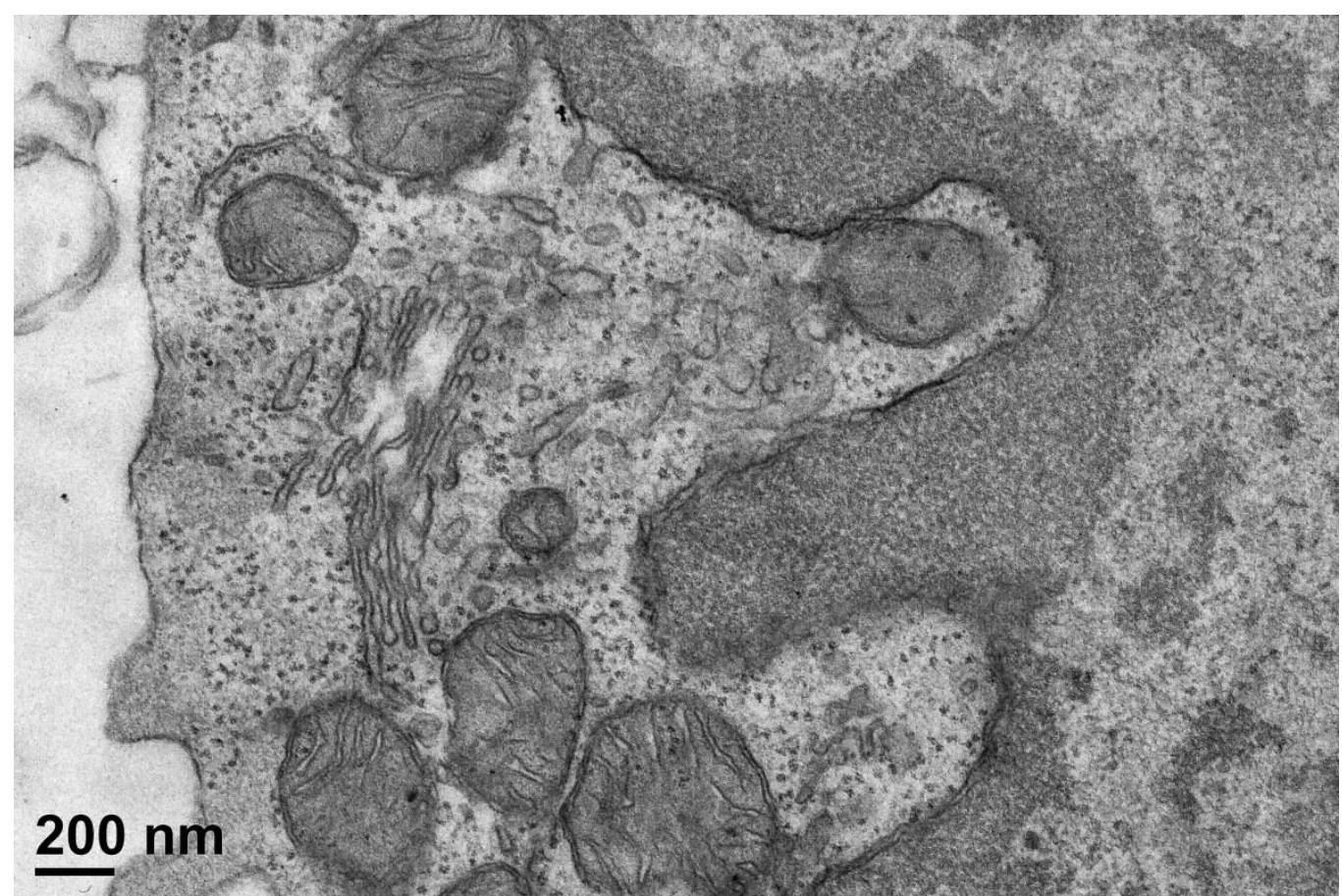

Figure 2. Figure 2: Higher magnification image of the cell showing subcellular structures. Scale bar: $200 \mathrm{~nm}$

\section{References}

[1] Knoll M, Ruska E. Das Elektronenmikroskop. Zeitschrift fur Phys. 1932;78:318-39.

[2] Knott G, Genoud C. Is EM dead? J Cell Sci. 2013;126:4545-52.

[3] Lidke DS, Lidke KA. Advances in high-resolution imaging - Techniques for three-dimensional imaging of cellular structures. J Cell Sci. 2012;125:2571-80. 